\title{
Quantum Hamiltonian formulation of the $q$-state Potts model : weak-coupling expansion
}

\author{
L. Turban \\ Laboratoire de Physique du Solide (*), Université de Nancy I, CO 140, F54037 Nancy, France
}

(Reçu le 27 octobre 1980, révisé le 16 février 1981, accepté le 19 février 1981)

\begin{abstract}
Résumé. - Une formulation quantique à une dimension du modèle de Potts à deux dimensions est étudiée en effectuant un développement en perturbation de l'énergie de l'état fondamental. Les séries donnant l'aimantation spontanée, la susceptibilité et la chaleur spécifique sont analysées par la méthode des approximants de Padé. Les exposants critiques, interpolés à la valeur exacte du couplage critique, sont en bon accord avec les conjectures de den Nijs, Alexander et Enting pour $q=3$ et $q=4$.

Abstract. - A one-dimensional quantum formulation of the two-dimensional $q$-state Potts model is studied using a weak-coupling perturbation expansion for the ground state energy. The series for the spontaneous magnetization, the susceptibility and the specific heat are analysed using the method of Pade approximants. The critical exponents, interpolated to the exact value of the critical coupling, are in good agreement with the conjectures of den Nijs, Alexander and Enting for $q=3$ and $q=4$.
\end{abstract}

1. Introduction. - Quantum Hamiltonian formulations in $d$-dimensions of classical spin systems in $d+1$-dimensions have been extensively studied in recent years. This method provided a new approach to the $2 \mathrm{~d}$ Ising problem which, as quantum counterpart, has the 1d Ising model in a transverse field solved by Pfeuty [1]. More recently other models have been studied among which planar vector models, $O(n)$ Heisenberg models and jauge models $[2,3]$. Since exact solutions are not known in these latter cases, various approximate methods were introduced, including real-space renormalization groups $[4,5]$, weak-coupling and strong-coupling expansions $[6,7]$, allowing the determination of phase diagrams and critical exponents identical to the classical exponents according to the universality hypothesis. An excellent review of these methods may be found in reference [2].

In this paper we study a quantum Hamiltonian formulation of the $q$-state Potts model [8], a generalization of the Ising model related to the percolation problem in the $q=1$ limit $[9,10]$ to the Ising model when $q=2$ and models of phase transitions in monolayers when $q=3$ and 4 [3]. An exact solution has been obtained by Baxter et al. [11] at the critical temperature on the square, honeycomb and trian-

(*) L.A. au C.N.R.S. no 155. gular lattices, using a correspondence with a sixvertex model. The transition was shown to be continuous when $q \leqslant 4$ whereas it is first-order above.

Den Nijs [12] proposed a relation between the $q$-state Potts model and eight-vertex model thermal exponents leading to :

$$
y_{\mathrm{T}}^{\mathrm{P}}=3 / 2[2+\pi /(\mu-\pi)] \quad \cos \mu=1 / 2 \sqrt{q}
$$

and according to Alexander [13], the $q=3$ Potts model belongs to the same universality class as the « hard hexagons model » recently solved by Baxter [14]. The $q=4$ Potts exponents have been conjectured by Enting [15].

This paper is organized as follows. First $(\S 2)$ we present a continuous time, discrete spatial lattice version of the 1d quantum Hamiltonian corresponding to the $2 \mathrm{~d}$ classical Potts model on the square lattice. The critical coupling of the quantum problem is deduced from the known critical line of the anisotropic classical problem. Then in $\S 3$ we develop a weak-coupling expansion for the ground state energy corresponding to the free energy of the statistical problem. The spontaneous magnetization, susceptibility and specific heat series are analysed using the method of Padé approximants. Since the diagrams were evaluated by hand, we limited ourselves to the fifth-order in the coupling constant $1 / \lambda$. The Padé analysis gives approximate values of the critical 
coupling $1 / \lambda_{\mathrm{c}}$ and the exponents $\beta_{q}, \gamma_{q}$ and $\alpha_{q}$. Although these approximate exponents suffer from a rather wide dispersion, they vary, to a good approximation, linearly with their corresponding critical couplings, allowing an interpolation to the known exact value

$$
1 / \lambda_{\mathrm{c}}=1 \text {. }
$$

This procedure provides accurate results in the cases $q=3$ and $q=4$. The spontaneous magnetization is exact in the Ising case, a result previously obtained by Hamer and Kogut [7].

2. Quantum Hamiltonian formulation. - In this section we make use of the now standard transfer matrix method developed by Fradkin and Susskind [16] for the Ising model in a transverse field to get the $\tau$-continuum, discrete lattice quantum Hamiltonian formulation of the $2 \mathrm{~d} q$-state Potts model. We have the following relations between both formulations [2] :

Statistical mechanics $(d+1)$ Quantum mechanics $(d)$

Free energy Ground state energy

Correlation functions Propagator

Reciprocal of the correla- Mass gap tion length

Transfer matrix. Evolution operator

Consider a square lattice with a Potts variable $\sigma(m)$ (which may lie in any one of its $q$-states) associated with each lattice site $m$. We will have to take strongly anisotropic interactions between the Potts variables in order to go to the $\tau$-continuum limit for the quantum Hamiltonian. One spatial direction of the statistical problem will play the role of the time axis in the quantum problem. Denote the unit vector in the temporal (spatial) direction by $\hat{\tau}(\hat{x})$. The action $S$ of the $q$-state Potts model reads $[8,10]$ :

$$
\begin{aligned}
S=-\sum_{m}\left[K_{\tau}\left(q \delta_{\sigma(m) \sigma(m+\hat{\tau})}-1\right)+\right. & \\
& \left.+K_{x}\left(q \delta_{\sigma(m) \sigma(m+\hat{x})}-1\right)\right]
\end{aligned}
$$

where $K_{\tau}\left(K_{x}\right)$ is the temporal (spatial) coupling. In order to construct the transfer matrix, it is convenient to introduce the Lagrangian for a pair of neighbouring rows in the temporal direction :

$$
\begin{aligned}
\mathfrak{L}(n, n+\hat{\tau})= & -q K_{\tau} \sum_{m}\left(\delta_{\sigma(m) s(m)}-1\right) \\
& -q K_{x} / 2 \sum_{m}\left(\delta_{\sigma(m) \sigma(m+\hat{x})}+\delta_{s(m) s(m+\hat{x})}\right)
\end{aligned}
$$

where $s(m)$ stands for $\sigma(m+\hat{\tau})$ (see Fig. 1). The sum runs over the $N$ sites of the rows in the spatial direction. The action may be rewritten as a sum over rows $S=\sum_{n} \mathfrak{L}(n, n+\hat{\tau})$ differing from equation (2) only by an unimportant constant term.

The transfer matrix between any two rows $n$ and $n+\hat{\tau}, T=\exp [-\mathfrak{L}(n, n+\hat{\tau})]$ is related to the

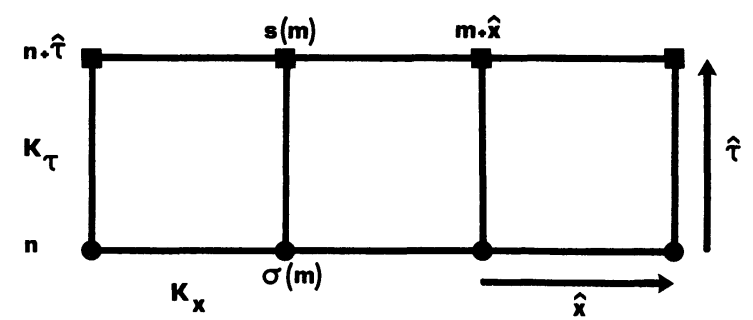

Fig. 1. - Spin variables on adjacent spatial rows of the twodimensional Potts model.

quantum mechanical evolution operator for an infinitesimal time step $\tau=i t$, involving the quantum Hamiltonian $\hat{H}$ through

$$
\hat{T}=\exp (-\tau \hat{H}) \approx 1-\tau \hat{H}+O\left(\tau^{2}\right) .
$$

It is easy to verify, through a study of the various matrix elements of the $\hat{T}$-matrix $[2,16]$, that the preceding expansion makes sense only when the extreme anisotropic limit

$$
K_{x} \rightarrow 0, \quad K_{\tau} \rightarrow \infty
$$

with

$$
K_{x}=\lambda \exp \left(-q K_{\tau}\right)
$$

is taken. The quantum Hamiltonian is then :

$\hat{H}=-q \lambda \sum_{m} \sum_{v=1}^{q} \hat{\pi}_{v}(m) \hat{\pi}_{v}(m+\hat{x})-\sum_{m} \sum_{v=1}^{q-1}\left[\hat{\sigma}_{1}(m)\right]^{v}$.

In order to write $\hat{H}$ in operator form, we associated with each site on the row $q$ orthonormal state vectors $|v\rangle(v=1,2, \ldots, q)$ defined modulo $q$, i.e.

$$
|v \pm q\rangle \equiv|v\rangle \quad(1 \leqslant v \leqslant q) .
$$

We introduced a flip-operator $\hat{\sigma}_{1}$ which, using bracket notations, reads :

$$
\hat{\sigma}_{1}=\sum_{v=1}^{q}|v\rangle\langle v-1|
$$

and which is a generalization for the $q$-dimensional basis set of the usual Pauli spin-flip operator. Acting on the state $|\alpha\rangle, \hat{\sigma}_{1}$ change it into the state $|\alpha+1\rangle$ $(\bmod . q)$. We also used products of $\hat{\sigma}_{1}$-operators

$$
\left(\hat{\sigma}_{1}\right)^{\mu}=\sum_{v=1}^{q}|v\rangle\langle v-\mu|
$$

and the projection operator

$$
\hat{\pi}_{v}=|v\rangle \cdot\langle v| \text {. }
$$

The following relations are easily derived :

$$
\begin{aligned}
& \left(\hat{\sigma}_{1}\right)^{q}=\hat{1} ; \quad \sum_{v=1}^{q} \hat{\pi}_{v}=\hat{1} ; \\
& \left(\hat{\sigma}_{1}\right)^{\mu}|\alpha\rangle=|\alpha+\mu\rangle \quad(\bmod . q) ; \\
& \hat{\pi}_{v}|\alpha\rangle=\delta_{v \alpha}|\alpha\rangle ; \quad \hat{\pi}_{v}\left(\hat{\sigma}_{1}\right)^{\mu}=\left(\hat{\sigma}_{1}\right)^{\mu} \hat{\pi}_{v-\mu} .
\end{aligned}
$$


Alternatively, one might use column vectors and matrix notations for which the relation with the Pauli operators is more apparent when $q=2$. For example, in the case $q=3$, the basis set is

$$
\left(\begin{array}{l}
1 \\
0 \\
0
\end{array}\right)\left(\begin{array}{l}
0 \\
1 \\
0
\end{array}\right)\left(\begin{array}{l}
0 \\
0 \\
1
\end{array}\right)
$$

and then

$$
\hat{\sigma}_{1}=\left(\begin{array}{lll}
0 & 0 & 1 \\
1 & 0 & 0 \\
0 & 1 & 0
\end{array}\right), \quad \hat{\pi}_{1}=\left(\begin{array}{lll}
1 & 0 & 0 \\
0 & 0 & 0 \\
0 & 0 & 0
\end{array}\right) .
$$

The generalization to higher $q$-values is straightforward.

The $q$-state Potts model is known to be self-dual [8] so that :

$$
\hat{H}(\lambda)=\lambda \hat{H}(1 / \lambda)
$$

and $\lambda_{\mathrm{c}}=1$.

Although not evident on equation (7), the selfduality has been proved recently in alternative quantum formulations making use of the $Z_{n}$ algebra by Kogut [17] and Horn et al. [18].

Alternatively $\lambda_{\mathrm{c}}$ may be deduced from the known expression for the anisotropic critical line of the $q$-state Potts model on the square lattice [19]

$$
\begin{aligned}
\exp \left[q\left(K_{x c}+K_{\tau c}\right)\right] & -\exp \left(q K_{x c}\right) \\
& -\exp \left(q K_{\tau c}\right)-(q-1)=0
\end{aligned}
$$

which in the appropriate limit $\left(K_{x} \rightarrow 0, K_{\tau} \rightarrow \infty\right)$ leads to $\lambda_{\mathrm{c}}=1$. In the Ising case, the spontaneous magnetization is known to be [20]

$$
M=\left(1-k_{\mathrm{r}}^{2}\right)^{1 / 8}
$$

where

$$
k_{\mathrm{r}}^{2}=\frac{16 Z_{x}^{2} Z_{\tau}^{2}}{\left(1-Z_{x}^{2}\right)^{2}\left(1-Z_{\tau}^{2}\right)^{2}}
$$

and

$$
Z_{i}=\exp \left(-2 K_{i}\right) \quad(i=x, \tau) .
$$

In the $\tau$-continuum limit we get

$$
M=\left[1-(1 / \lambda)^{2}\right]^{1 / 8} .
$$

3. Weak-coupling expansion. - Let us introduce the operator $\left({ }^{1}\right)$

with

$$
W(h)=W_{0}(h)-(1 / \lambda) V
$$

$$
\begin{array}{r}
W_{0}(h)=q \sum_{m}\left[1-\sum_{v=1}^{q} \pi_{v}(m) \pi_{v}(m+\hat{x})\right]- \\
-h \sum_{m}\left(q \pi_{1}(m)-1\right)
\end{array}
$$

( $\left.{ }^{1}\right)$ From now on we shall omit the « hats " on the operators.

$$
V=\sum_{m} \sum_{\nu=1}^{q-1}\left[\sigma_{1}(m)\right]^{v}
$$

so that $W(0)=(1 / \lambda) H$. An external field coupled to the order parameter have been added in equation $(14 b)$ in order to fix the ground state $|0\rangle$ of $W_{0}(h)$ $\left(\pi_{1}(m)|0\rangle=|0\rangle\right.$, all the sites lying in the state $|v\rangle=|1\rangle)$.

The magnetization per site $M(h)$, according to the Feynman-Hellmann theorem, will be given by :

$$
M(h)=-\frac{1}{N(q-1)} \frac{\partial E_{0}(h)}{\partial h}
$$

where $E_{0}(h)$ is the ground state energy of $W(h)$ corresponding to the free energy in the thermal problem. The zero field susceptibility reads

$$
\chi=\left.\frac{\partial M(h)}{\partial h}\right|_{h=0}
$$

and the singular part of the specific heat may be studied on [7]

$$
C=-\frac{1}{N(q-1)} \frac{\partial^{2} E_{0}}{\partial(1 / \lambda)^{2}} .
$$

$E_{0}(h)$ have been calculated using a RayleighSchrödinger perturbation expansion up to terms of order $(1 / \lambda)^{5}$ :

$$
E_{0}(h)=\sum_{n} \varepsilon_{n}(-1 / \lambda)^{n}
$$

with

$$
\begin{aligned}
\varepsilon_{0} & =-N h(q-1) \\
\varepsilon_{1} & =0 \\
\varepsilon_{2} & =\langle 0|V g V| 0\rangle \\
\varepsilon_{3} & =\langle 0|V g V g V| 0\rangle \\
\varepsilon_{4} & =\langle 0|V g V g V g V| 0\rangle-\varepsilon_{2}\left\langle 0\left|V g^{2} V\right| 0\right\rangle \\
\varepsilon_{5}= & \langle 0|V g V g V g V g V| 0\rangle- \\
& \quad-\varepsilon_{2}\left\langle 0\left|V g^{2} V g V+V g V g^{2} V\right| 0\right\rangle \\
& \quad-\varepsilon_{3}\left\langle 0\left|V g^{2} V\right| 0\right\rangle
\end{aligned}
$$

where $g$ is the resolvent

$$
g=\frac{1-|0\rangle\langle 0|}{\varepsilon_{0}-W_{0}(h)} .
$$

The $\varepsilon_{n}$ are evaluated using a diagrammatic technique (Fig. 2). A $n$th order graph has $n$ vertices corresponding to the action of the perturbation $V$ on a given site. A site initially in the ground state may be flipped into any one of the $(q-1)$ other states. A flipped state is pictured by a vertical line. In an intermediate state, one has at least one flipped state; otherwise the matrix element of $g$ vanishes. In the final state all the sites must be back in the ground state of $W_{0}(h)$ in order to get a nonvanishing contribution. As a 


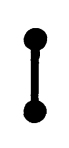

$\mathbf{a}$

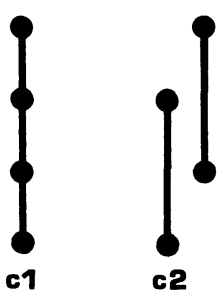

Fig. 2. - Diagrams contributing to $E_{0}(h)$ in the 2nd $(a)$, 3rd $(b)$ and 4 th order $(c)$ in $1 / \lambda$. The vertical lines picture a flipped state; the black circles represent the action of the perturbation $\hat{V}$. Horizontal dotted lines join flipped states on nearest-neighbour sites.

result the first-order term $\varepsilon_{1}$ is zero. In the diagrams of the 4th or higher orders, one must sum over the ordering of the vertices on different sites and take care of the fact that two flipped states may be or not on nearest-neighbour sites. When there is a single flip in the intermediate state $\langle g\rangle=-(2 q+h q)^{-1}$.
With two flips on distant sites $\langle g\rangle=-(4 q+2 h q)^{-1}$; whereas on two neighbouring sites

$$
\langle g\rangle=-(2 q+2 h q)^{-1}
$$

when the state is the same on both and

$$
\langle g\rangle=-(3 q+2 h q)^{-1}
$$

for different states.

Figure $2 a$ gives the second-order contribution

$$
\varepsilon_{2}=-\frac{N(q-1)}{2 q+h q}
$$

and figure $2 b$ leads to

$$
\varepsilon_{3}=\frac{N(q-1)(q-2)}{(2 q+h q)^{2}}
$$

for the third-order term. The factor $(q-1)$ comes from the $(q-1)$ possible final states for the first flip whereas there remains only $(q-2)$-states other than the ground state for the second flip. This contribution as well as all other odd contributions vanishes for the Ising model $(q=2)$. Using these rules we get the ground state energy

$$
\begin{aligned}
& \frac{E_{0}(h)}{N(q-1)}=-h-\frac{(1 / \lambda)^{2}}{2 q+h q}-\frac{(q-2)(1 / \lambda)^{3}}{(2 q+h q)^{2}}- \\
& -\left[\frac{4(q-2)}{3 q+2 h q}+\frac{2}{q+h q}+\frac{q^{2}-7 q+7}{2 q+h q}\right] \frac{(1 / \lambda)^{4}}{(2 q+h q)^{2}} \\
& -\left[\frac{q^{2}-13 q+13}{(2 q+h q)^{2}}+\frac{8}{2 q+h q}\left(\frac{1}{2 q+2 h q}+\frac{q-2}{3 q+2 h q}\right)+\frac{8}{3 q+2 h q}\left(\frac{1}{q+h q}+\frac{q-3}{3 q+2 h q}\right)\right] \frac{(q-2)(1 / \lambda)^{5}}{(2 q+h q)^{2}} .
\end{aligned}
$$

From equations $(15,16,17,22)$ we deduce perturbation expansions for the spontaneous magnetization

$$
M(0)=\sum_{n} a_{n}(1 / i)^{n},
$$

the susceptibility $\chi=\sum_{n} b_{n}(1 / \lambda)^{n}$ and the specific heat $C=\sum_{n} c_{n}(1 / \lambda)^{n}$. The expansion coefficients are given in table $I$.

Near the critical coupling $1 / \lambda_{\mathrm{c}}=1$,

$$
M(0) \sim\left(1 / \lambda_{c}-1 / \lambda\right)^{\beta_{q}}
$$

and

$$
\frac{\partial \ln M}{\partial(1 / \lambda)}=\frac{-\beta_{q}}{1 / \lambda_{\mathrm{c}}-1 / \lambda} .
$$

The series expansion for $\partial \ln M / \partial(1 / \lambda)$ may be used to get the coefficients of the Padé approximant

$$
\mathfrak{T}_{N}^{D}(1 / \lambda)=\frac{n_{0}+n_{1}(1 / \lambda)+\cdots+n_{N}(1 / \lambda)^{N}}{1+d_{1}(1 / \lambda)+\cdots+d_{D}(1 / \lambda)^{D}}
$$

The approximate critical coupling is a pole of $\mathcal{T}_{N}^{D}(1 / \lambda)$ and the corresponding residue gives $-\beta_{q}$. The same analysis has been carried out for $\gamma_{q}$ and $\alpha_{q}$. The results are given on table II.

4. Discussion. - The Padé approximants provide approximate critical couplings ranging from about 0.5 to 1.5 . This is due to the small number of terms we took into account in the series expansions. To a good approximation, we get a linear variation of the exponents with the approximate critical couplings (Fig. 3); this is particularly true when $q=3$ and 4 . This enables us to interpolate the exponents to their values at the known exact critical coupling $1 / \lambda_{c}=1$. The results are given in table III. 
Table I. - Coefficients of the weak-coupling expansions for the spontaneous magnetization (a), the susceptibility (b) and the specific heat (c).

\begin{tabular}{|c|l|l|l|l|}
\hline$n$ & \multicolumn{1}{|c|}{1} & 2 & 3 & 4 \\
\hline 0 & 1 & 1 & 1 & 1 \\
\hline 1 & 0 & 0 & 0 & 0 \\
\hline 2 & -0.25 & -0.125 & -0.0833333 & -0.0625 \\
\hline 3 & 0.25 & 0 & -0.0277778 & -0.03125 \\
\hline 4 & -0.6319444 & -0.0546875 & -0.0228909 & -0.0183377 \\
\hline 5 & 1.3935185 & 0 & -0.0200617 & -0.0165292 \\
\hline
\end{tabular}

(a)

\begin{tabular}{|l|l|l|l|l|}
\hline$n$ & \multicolumn{1}{|c|}{1} & 2 & 3 & 4 \\
\hline 0 & 0 & 0 & 0 & 0 \\
\hline 1 & 0 & 0 & 0 & 0 \\
\hline 2 & 0.25 & 0.125 & 0.0833333 & 0.0625 \\
\hline 3 & -0.375 & 0 & 0.0416667 & 0.046875 \\
\hline 4 & 1.8842593 & 0.203125 & 0.0783608 & 0.0524450 \\
\hline 5 & -5.3498178 & 0 & 0.0825607 & 0.0647945 \\
\hline
\end{tabular}

(b)

\begin{tabular}{|c|l|l|l|l|}
\hline$n$ & \multicolumn{1}{|c|}{1} & \multicolumn{1}{|c|}{2} & 3 & 4 \\
\hline 0 & 1 & 0.5 & 0.3333333 & 0.25 \\
\hline 1 & -1.5 & 0 & 0.1666667 & 0.1875 \\
\hline 2 & 3.5 & 0.1875 & 0.0925926 & 0.1015625 \\
\hline 3 & -9.0277778 & 0 & 0.1080247 & 0.0965712 \\
\hline
\end{tabular}

(c)

Table III. - Critical exponents interpolated (when possible) to the exact value of the critical coupling (a) compared to the exact values (b), series results and conjectured values.

\begin{tabular}{|c|c|c|c|c|}
\hline$q$ & 1 & 2 & 3 & 4 \\
\hline$\beta_{q}$ & $\begin{array}{c}0.179(a) \\
0.138 \pm 0.007[21]\end{array}$ & $0.125(a, b)$ & $\begin{array}{l}0.104(a) \\
0.111[14]\end{array}$ & $\begin{array}{l}0.091(a) \\
0.083[15]\end{array}$ \\
\hline$\gamma_{q}$ & $2.43 \pm 0.03[21]$ & $\begin{array}{l}1(a) \\
1.75(b)\end{array}$ & $\begin{array}{l}1.354(a) \\
1.444[14]\end{array}$ & $\begin{array}{l}1.062(a) \\
1.167[15]\end{array}$ \\
\hline$\alpha_{q}$ & $-0.667[12]$ & $0[12 b]$ & $\begin{array}{l}0.410(a) \\
0.333[12,14]\end{array}$ & $\begin{array}{l}0.526(a) \\
0.667[12,15]\end{array}$ \\
\hline
\end{tabular}

Table II. - Magnetization exponents $\beta_{q}(\mathrm{a})$, susceptibility exponents $\gamma_{q}(\mathrm{~b})$, specific heat exponents $\alpha_{q}$ (c) and critical couplings $1 / \lambda_{\mathrm{c}}$ deduced from the Padé analysis of the series.

\begin{tabular}{|c|c|c|c|c|c|c|c|c|}
\hline$(N, D)$ & $1 / \lambda_{\mathrm{c}}$ & $\beta_{1}$ & $1 / \lambda_{\mathrm{c}}$ & $\beta_{2}$ & $1 / \lambda_{\mathrm{c}}$ & $\beta_{3}$ & $1 / \lambda_{\mathrm{c}}$ & $\beta_{4}$ \\
\hline$(1,1)$ & - & - & - & - & - & - & 1.333 & 0.222 \\
\hline$(1,2)$ & 0.868 & 0.114 & 1 & 0.125 & 1.090 & 0.136 & 1.174 & 0.154 \\
\hline$(2,1)$ & - & - & - & - & 0.790 & 0.041 & 1.155 & 0.144 \\
\hline$(1,3)$ & - & - & 1 & 0.125 & 0.973 & 0.095 & 0.984 & 0.084 \\
\hline$(2,2)$ & 1.394 & 0.372 & 1 & 0.125 & 0.923 & 0.075 & 1.448 & 0.252 \\
\hline$(3,1)$ & - & - & - & - & 0.943 & 0.083 & 0.878 & 0.048 \\
\hline
\end{tabular}

(a)

\begin{tabular}{|c|c|c|c|c|c|c|c|c|}
\hline$(N, D)$ & $1 / \lambda_{\mathrm{c}}$ & $\gamma_{1}$ & $1 / \lambda_{\mathrm{c}}$ & $\gamma_{2}$ & $1 / \lambda_{\mathrm{c}}$ & $\gamma_{3}$ & $1 / \lambda_{\mathrm{c}}$ & $\gamma_{4}$ \\
\hline$(0,2)$ & 0.483 & 0.847 & 0.784 & 1 & 0.998 & 1.143 & 1.163 & 1.279 \\
\hline$(1,1)$ & - & - & - & - & 0.307 & 0.153 & 0.672 & 0.504 \\
\hline$(0,3)$ & - & - & 0.784 & 1 & 0.836 & 0.973 & 0.896 & 1.004 \\
\hline$(1,2)$ & 0.711 & 1.114 & 0.784 & 1 & 0.763 & 0.803 & 0.679 & 0.514 \\
\hline$(2,1)$ & - & - & - & - & 0.967 & 1.524 & 0.679 & 0.514 \\
\hline
\end{tabular}

(b)

\begin{tabular}{|c|c|c|c|c|}
\hline$(N, D)$ & $1 / \lambda_{\mathrm{c}}$ & $\alpha_{3}$ & $1 / \lambda_{\mathrm{c}}$ & $\alpha_{4}$ \\
\hline$(0,1)$ & 1.636 & 0.818 & - & - \\
\hline$(0,2)$ & 0.743 & 0.240 & 0.940 & 0.418 \\
\hline$(1,1)$ & 0.449 & 0.062 & 0.375 & 0.035 \\
\hline
\end{tabular}

(c)

Although the estimates are poor for the percolation problem $(q=1), \beta$ and $\lambda_{\mathrm{c}}$ are exact for the Ising model $(q=2)$. The $q=3$ values are consistent with the exact results for the " hard hexagons problem " [14] $(\alpha \approx 0.333, \beta \approx 0.111, \gamma \approx 1.444)$ which, according to a conjecture of Alexander [13], belongs to the same universality class. A good agreement with a conjecture of Enting [15] giving $\alpha \approx 0.667, \beta \approx 0.083$ and $\gamma \approx 1.167$, is found when $q=4$. 


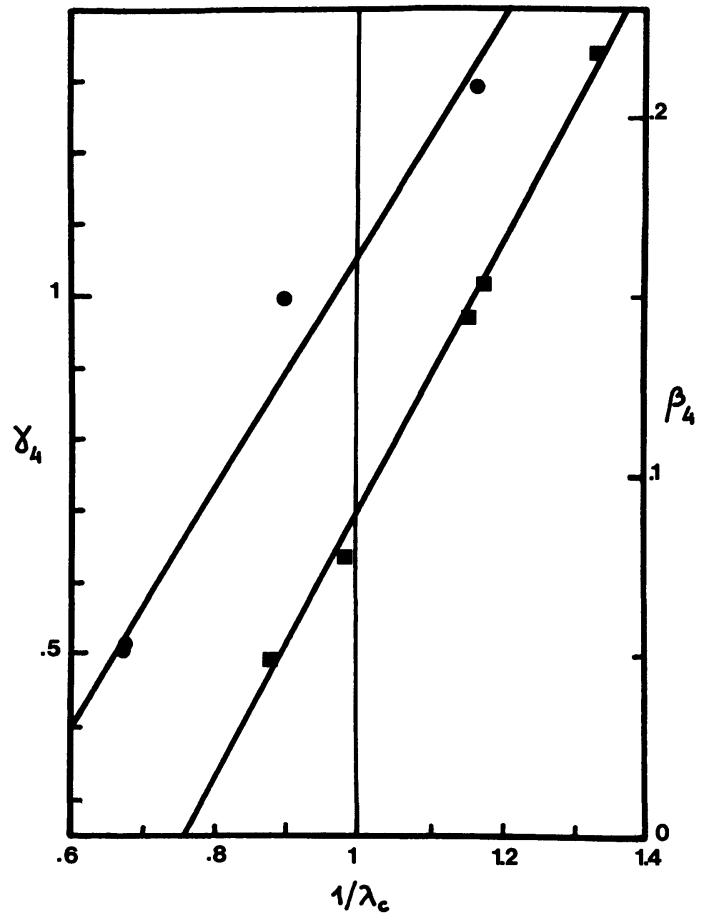

Fig. 3. - Variation of the critical exponents $\beta$ (squares) and $\gamma$ (circles) with the approximate critical coupling $1 / \lambda_{\mathrm{c}}$ when $q=4$. The heavy lines are least-square fits of the results assuming a linear variation.

Since the best estimates are obtained for $\beta_{q}$, we used the best Padé approximants in each case to calculate the spontaneous magnetization

$$
M(1 / \lambda)=\exp \left[\int_{0}^{1 / \lambda} \mathfrak{T}_{N}^{D}(x) \mathrm{d} x\right] .
$$

\section{References}

[1] Pfeuty, P., Ann. Phys. (NY) 57 (1970) 79.

[2] Kogut, J. B., Rev. Mod. Phys. 51 (1979) 659.

[3] Barber, M., Phys. Rep. 59 (1980) 375.

[4] Jullien, R., Pfeuty, P., Fields, J. N. and Doniach, S., Phys. Rev. B 18 (1978) 3568.

[5] Drell, S. D., Weinstein, M. and Yankielovicz, S., Phys. Rev. D 14 (1976) 487.

[6] Hamer, C. J., Kogut, J. B. and Susskind, L., Phys. Rev. Lett. 41 (1978) 1337.

[7] Hamer, C. J. and Kogut, J. B., Phys. Rev. B 20 (1979) 3859.

[8] Potrs, R. B., Proc. Cambridge Philos. Soc. 48 (1952) 106.

The Potts model belongs to the same universality class as the $Z_{n}$ model when $q=2$ and 3 . The $Z_{n}$ model corresponds to two noninteracting Ising models when $n=4$ and has two phase transitions when $n \geqslant 5$ in 2D. A quantum formulation of the $Z_{n}$ model has been recently studied by S. Elitzur, R. B. Pearson and J. Shigemitsu, Phys. Rev. D 19 (1979) 3698 and C. J. Hamer and J. B. Kogut, Phys. Rev. B 22 (1980) 3378.

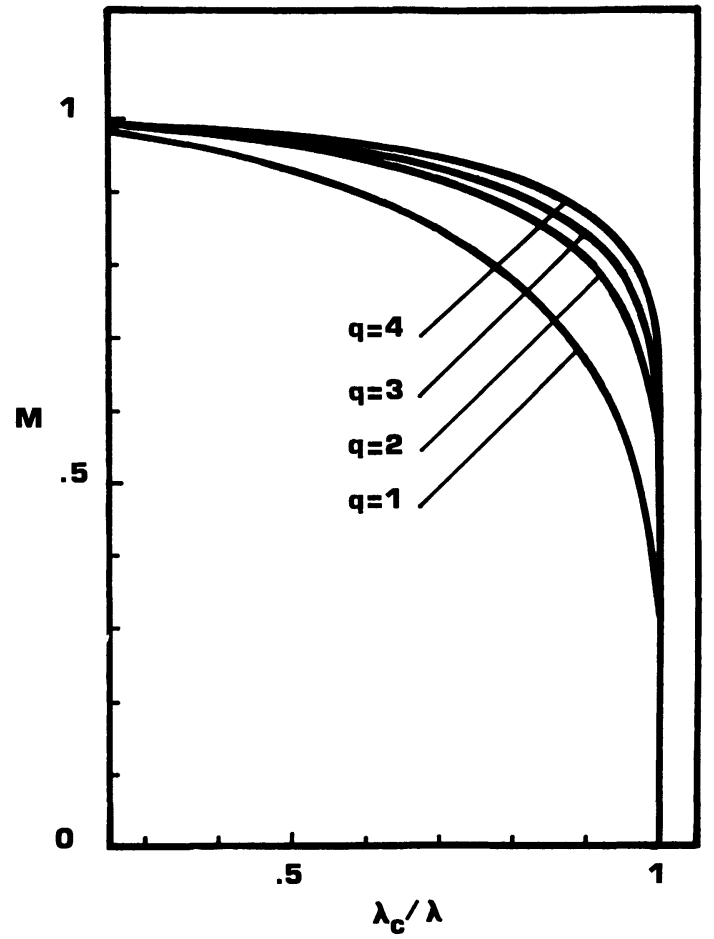

Fig. 4. - Spontaneous magnetization deduced from the best Padé approximants (Eq. (25) in the text).

When $q=2$, we recover the exact result of equation (13). $M\left(\lambda_{\mathrm{c}} / \lambda\right)$ (Fig. 4) behaves as expected as a function of $q$, the transition becoming steeper with increasing $q$.
[9] Kasteleyn, P. W. and Fortuin, C. M., J. Phys. Soc. Japan Suppl. 26 (1969) 11.

[10] Wu, F. Y., J. Stat. Phys. 18 (1978) 115.

[11] Bayter, R. J., Temperley, H. N. V. and Ashley, S. E., Proc. R. Soc. London A 358 (1978) 535.

[12] Den Niss, M. P. M., J. Phys. A 12 (1979) 1857.

[13] Alexander, S., Phys. Lett. 54A (1975) 353.

[14] Baxter, R. J., J. Phys. A 13 (1980) L-61.

[15] Enting, I. G., J. Phys. A 8 (1975) L-35.

[16] Fradkin, E. and Susskind, L., Phys. Rev. D 17 (1978) 2637.

[17] Kogut, J. B., Phys. Rep. 67 (1980) 67 and references therein.

[18] Horn, D., Karliner, M. and Yankielowicz, S., Nucl. Phys. B 170 (1980) 467. See also Hu, B., J. Phys. A 13 (1980) L-321.

[19] See for example Burkardt, T. W. and Southern, B. W., J. Phys. A 11 (1978) L-247.

[20] Syosı, I., Phase transitions and critical phenomena, Vol. I, C. Domb and M. S. Green eds. (London : Academic Press) 1972, p. 269.

[21] Gaunt, D. S. and Sykes, M. F., J. Phys. A 9 (1976) 1109. 